\title{
RE: Breast Cancer Mortality After Implementation of Organized Population-Based Screening in Norway
}

\author{
Per-Henrik Zahl, DrMedSci*
}

Norwegian Institute of Public Health, Domain for Mental and Physical Health, Skøyen, Oslo, Norway

${ }^{*}$ Correspondence to: Per-Henrik Zahl, DrMedSci, Norwegian Institute of Public Health, Domain for Mental and Physical Health, PO Box 222, Skøyen N-0213, Oslo, Norway (e-mail: per-henrik.zahl@fhi.no).

Sebuødegård et al. (1) report that public mammography screening has reduced breast cancer mortality by $20 \%$ and other factors reduced mortality by $23 \%$ in the age group $50-88$ years in Norway.

The authors excluded all women younger than 50 years from their analyses. Women in Norway younger than 50 years are not invited for screening but have experienced a considerably larger reduction in breast cancer mortality since 1989 (-35.2\%) than women aged 50-69 years (-22.6\%) who are invited (2). Improved treatment and centralized care may thus explain the entire observed reduction in breast cancer mortality also in the screened age groups. Effects of comparatively small differences in treatment standard between regions, which this and other observational studies cannot account for, can therefore be wrongly ascribed to screening. Furthermore, the results of this study would suggest that the effect of modern treatment is substantially reduced after age 50 years, which is not supported by the evidence (3)

There is a problem with the model assumptions: according to the authors, "The rate ratio (RR) of $\mathrm{BC}$ mortality between the non-invited and pseudo-non-invited women was interpreted as the change in BC mortality over time due to BC treatment, awareness and care (treatment effect). This effect was assumed to be linear over time," but during the period 1977-1995, the breast cancer mortality rate was almost constant, and then it started falling from 1995 (2). The rate ratio is not linear over time.

Public mammography screening started in 1996 and was rolled out nationally over the following decade (1). Women invited to mammography were included 5.6years later, on average (my calculation) than women in the noninvited group, and the follow-up time was much shorter in the noninvited group (average follow-up time is 4.1 years in the noninvited period and 10.4 years in the invited period) (1), because noninvited women were censored during the period 2000-2005. This time difference probably has little effect in the prescreening period where there is no public screening and very little adjuvant therapy. However, it may have a huge effect in the screening period. For example, many women in the invited group were offered trastuzumab in the period 2005-2014 page 84 (4) and page 63 (5), respectively, but hardly any in the noninvited group. There is also a major difference in the use of the centennial node operation technique between the groups (6). Thus, the study design is flawed because treatment effects after 1996 are not comparable in the 2 groups.

\section{Notes}

Disclosures: The author declares no disclosures.

\section{References}

1. Sebuødegård S, Botteri E, Hofvind S. Breast cancer mortality after implementation of organized population-based screening in Norway. J Natl Cancer Inst. 2020;112(8):djz220. https://doi.org/10.1093/jnci/djz220.

2. Autier P, Boniol M, La Vecchia C, et al. Disparities in breast cancer mortality trends between 30 European countries: retrospective trend analysis of WHO mortality database. BMJ. 2010;341(Aug11 1):c3620.

3. Early Breast Cancer Trialists' Collaborative Group (EBCTCG). Aromatase inhibitors versus tamoxifen in early breast cancer: patient-level metaanalysis of the randomised trials. Lancet. 2015;386(10001):1341-1352. doi: 10.1016/S0140-6736(15)61074-1.

4. Norwegian Institute of Public Health. Drug Consumption in Norway 2003-2007. Oslo, Norway: Norwegian Institute of Public Health; 2008.

5. Norwegian Institute of Public Health. Drug Consumption in Norway 2010-14. Oslo, Norway: Norwegian Institute of Public Health; 2015.

6. Kalager M, Kåresen R, Wist E. Fylkesvise forskjeller i overlevelse av brystkreft. Tidsskriftet 2009;129(24):2595-2600. 